SISISOMOS VolumenXninmero2,

AMERICANOS

Revista de Estudios

Transfronterizos

julio-diciembrede2020.

Recibido: 10 de septiembre de 2019.

Aprobado: 13 de septiembre de 2020.

\title{
Las políticas migratorias en México ante el cambio de gobierno
}

(2018-2024)*

\section{Migration policies in Mexico with respect to the change of government}

(2018-2024)

\author{
Rodolfo García Zamora ** \\ Selene Gaspar Olvera*** \\ Universidad Autónoma de Zacatecas, México. \\ Pascual García Macías ${ }^{* * * *}$ \\ Universidad Técnica Particular de Loja, Ecuador.
}

\begin{abstract}
* El artículo se inscribe en una investigación titulada "Las políticas de desarrollo económico y migración en México 2018 a 2024", llevada a cabo en el doctorado en Estudios del Desarrollo de la Universidad Autónoma de Zacatecas, México.

** Doctor en Ciencias Económicas, Universidad Autónoma de Barcelona, España. Docente-investigador de la Unidad Académica en Estudios del Desarrollo, Universidad Autónoma de Zacatecas, México. Correo electrónico: rgarciazamora54@gmail.com, ORCID: https://orcid.org/0000-0002-0685-3165

*** Maestra en Demografía Social y Actuaría, UNAM. Investigadora de la Unidad Académica en Estudios del Desarrollo, Universidad Autónoma de Zacatecas, adscrita al Proyecto Sistema de Información Sobre Migración y Desarrollo (SIMDE-UAZ), Ciudad de México, México. Correo electrónico: selene.gasparolvera@gmail.com, ORCID: https://orcid.org/0000-0002-1312-5385

**** Doctor en Movilidad Humana, Universidad de Valencia, España. Profesor investigador a tiempo completo, Universidad Técnica Particular de Loja, Ecuador. Correo electrónico: pasgegar84@gmail.com, ORCID: https://orcid.org/0000-0001-8368-0811
\end{abstract}

Cómo citar este artículo: García, R., Gaspar, S. y García, P. (2020) Las políticas migratorias en México ante el cambio de gobierno (2018-2024). Si Somos Americanos. Revista de Estudios Transfronterizos, 20(2), 186-208. doi: $10.4067 / \mathrm{S} 0719-09482020000200186$

(c) (1) Este es un artículo de acceso abierto bajo licencia Creative Commons Atribución 4.0 Internacional 


\title{
Resumen
}

En las últimas cuatro décadas, la migración internacional de mexicanos a Estados Unidos se convirtió en una válvula de escape a los problemas económicos, sin que el Gobierno mexicano manifestase preocupación por el diseño de políticas públicas de desarrollo económico, migración y derechos humanos que incidan en las causas estructurales de los flujos migratorios. Las políticas sobre migración fueron parciales, reactivas y tardías, y forman parte de las políticas de seguridad del Estado mexicano. Estas no consideraron la causa estructural de las mismas, ni los derechos humanos de todos los migrantes que salían,

llegaban, pasaban y retornaban a México. Ante este panorama, el nuevo Gobierno mexicano enfrenta el doble reto de construir y aplicar una nueva estrategia de desarrollo nacional, soberana, con empleo, equidad y seguridad humana para todos y, al mismo tiempo, la necesidad de incluir una política de Estado que involucre el desarrollo integral, la migración y los derechos humanos, la cual contemple las siete dimensiones migratorias del país como espacio de origen, tránsito, destino, retorno, desplazamientos, asilo y refugio. Asimismo, que integre la migración proveniente de Centroamérica como parte importante de la misma, en razón de la trascendencia creciente que tiene ya en varias de esas dimensiones en los diferentes flujos poblacionales del país.

Palabras clave: políticas migratorias, migración, neoliberalismo.

\begin{abstract}
For more than forty years, the international migration of Mexicans to the United States has represented an escape valve in terms of economic problems. However, there was no concern on the part of previous Mexican governments to design public policies for economic development, migration and human rights that could impact the structural causes of such migratory flows. Rather, migration policies have been partial, reactive and overdue, while also forming part of the security policies of the Mexican State. Moreover, they have not considered the structural causes of migration, nor the human rights of all migrants who have left, arrived, passed through or returned to Mexico. The new Mexican government has thus faced the double challenge of creating and implementing a new national, sovereign, employment, equity and human security development strategy for all. This has also been accompanied by a State policy on comprehensive development, migration and human rights that responds to the seven migratory dimensions of the country as a place of origin, transit, destination, return, the increasing displacement of migrants, asylum and refuge. Furthermore, an important component is the integration of Central American migration due to the increasing importance this already has in various dimensions of the different population flows taking place in the country.
\end{abstract}

Keywords: migration policies, migration, neoliberalism. 


\section{Introducción}

Frente a la compleja crisis migratoria que experimenta México actualmente, adquieren gran relevancia las propuestas de política migratoria que han hecho las organizaciones migrantes mexicanas en los últimos diez años sin apoyo real del gobierno. En el discurso del nuevo Gobierno, este plantea que solo el desarrollo puede reducir las migraciones junto con políticas migratorias con enfoque de derechos humanos. Esto esboza un triple desafío: que el gobierno construya realmente políticas de desarrollo económico, migración y derechos humanos; que los migrantes fortalezcan sus propuestas en la agenda nacional; y que se cuente con el respaldo legal, técnico y presupuestal para gestionar esas políticas con los cambios institucionales necesarios.

Durante más de cuatro décadas, México se convirtió en una enorme fábrica de pobres y migrantes, cuya dinámica económica ha dependido de las exportaciones de la maquila electrónica y el ensamble automotriz. Estructuralmente, la incapacidad de generación de empleo suficiente y bien remunerado alimentó la migración creciente a Estados Unidos durante su largo periodo de crecimiento económico hasta la crisis económica de 2007-2009. Previo a esta ocurrió la contracción económica de 2001 y el ataque a las Torres Gemelas, en ese mismo año, cuyos acontecimientos generaron un cambio en la tendencia de la migración mexicana a Estados Unidos, reduciendo los flujos emigratorios por un largo periodo (Gaspar, 2012, 2018). La Organización Internacional para las Migraciones (OIM, 2009) señala que "las crisis financieras tienen un impacto en la migración y el desarrollo que se manifiesta en la reducción de las remesas, en la aceleración de la migración altamente calificada y en la ocurrencia de retornos a gran escala" (p. 1). Por esto es que los países emisores de migrantes deben diseñar políticas de protección a sus migrantes en el extranjero y prepararse para la reintegración de quienes regresan, puesto que la estrategia de exportación de mano de obra puede tener consecuencias no deseadas, tal como ha ocurrido en México.

En efecto, los impactos de las crisis económicas de Estados Unidos de 2001 y la de 20072009 sobre el mercado laboral y las políticas de seguridad nacional, que se intensificaron con el ataque terrorista de 2001 sobre la migración internacional con mayores sanciones y deportaciones crecientes, provocaron cambios importantes en el sistema migratorio de México y Estados Unidos. Fue así como el inicio del siglo XXI se caracterizó por un flujo creciente de migración irregular cercano al medio millón en los años previos a la crisis señalada (Canales, 2012), factores que en los últimos 15 años han generado un nuevo contexto político y económico en el cual es posible enmarcar las nuevas tendencias de la migración México- Estados Unidos (Canales y Meza, 2016).

Las crisis recurrentes experimentadas por la nación vecina de México precarizan aún más el mercado laboral estadounidense y las condiciones laborales de quienes aún cuentan con él. En este contexto, los más afectados son los trabajadores de menor calificación y en 
condición indocumentada de residencia, quienes enfrentan la reducción del tiempo de trabajo y de sus salarios, migrantes que finalmente tienen mayor tendencia al retorno (García, 2019; García y Gaspar 2017).

Con los impactos de la crisis económica de Estados Unidos reflejados en la inmigración de mexicanos y en el desplome del crecimiento económico de México, se percibe no solo la enorme vulnerabilidad de la economía mexicana para crecer y generar los empleos necesarios, sino también para poder emplear y reintegrar a los millones de deportados y retornados que se suman a los 52,4 millones de personas en el país en condición de pobreza en 2018, situación que con la pandemia se agrava e incrementa el número de pobres y por ingresos y la pobreza laboral. Esta última se estima aumentará de 37,3\% a 45,8\% de la población en los primeros dos trimestre de 2020 (CONEVAL, 2020). La disfuncionalidad no solo se presenta en la estructura económica nacional, también en el marco normativo sobre población y migración que resulta obsoleto para normar las múltiples dimensiones migratorias del país como espacio de origen, tránsito, destino, retorno y desplazamiento internos crecientes.

\section{Planes y programas de desarrollo para migrantes en México}

En el contexto anterior de profunda crisis económica, social y de seguridad en México, aunado a la pandemia, planteábamos que era apremiante una reorientación del modelo económico y de la gestión gubernamental para recuperar la paz del país, la cohesión social y la credibilidad de los ciudadanos en las instituciones públicas, colocando en el centro la generación de empleo y la seguridad humana para toda la población. Señalábamos que el reto para México y los demás países latinoamericanos de alta migración internacional radica en construir verdaderas políticas de Estado que impulsen el desarrollo económico y atiendan el problema de la migración, que sean integrales y de largo plazo y que fortalezcan el mercado interno, construyendo nuevos proyectos de desarrollo económico nacional, con estrategias específicas para los diferentes sectores económicos y regiones. Estas políticas deben generar los empleos necesarios para hacer viable el que todos los latinoamericanos puedan ejercer su derecho a no emigrar, es decir, que a mediano plazo la emigración sea una opción más para vivir mejor y no una necesidad, como sucede hasta ahora (García, 2010).

Ante la presión creciente ejercida en gran medida por los deportados de Estados Unidos en los primeros años posteriores a la crisis de 2008 y por el incremento de la transmigración de migrantes centroamericanos por México rumbo a Estados Unidos, en 2010 se inició un debate nacional sobre una nueva Ley de Migración y su reglamento, que llevó más de dos años, promovida en gran medida por 83 organizaciones de la sociedad civil. Estas, aprovechando la experiencia de interlocución e incidencia con el Estado mexicano para ese objetivo, se constituyeron como Colectivo Plan Nacional de Desarrollo-Migración con el 
fin de elaborar la Agenda Estratégica Transnacional de Migración para el Plan Nacional de Migración y Desarrollo 2013-2018. Los investigadores Marco Castillo y John Burstein explican los objetivos principales de la Agenda Estratégica Transnacional:

1. Articulación de una visión integral y multidimensional de la relación entre migración, desarrollo y seguridad humana con los mecanismos que integren la migración de forma transversal en el Plan Nacional de Desarrollo 2013-2018.

2. Incorporación explícita del fenómeno migratorio, la vinculación de la migración y desarrollo, y de las funciones y obligaciones de la administración pública federal para garantizar derechos en un sistema nacional de rendición de cuentas, con sustento en indicadores de gestión, acceso amplio y exhaustivo a información en materia migratoria, mecanismos de control eficaz y sanciones claras.

3. Creación de una estructura de coordinación interinstitucional y entre los tres órdenes de gobierno en materia migratoria, en la que participen la ciudadanía, organizaciones de la sociedad civil y académicos.

4. Creación de una estrategia nacional para la protección, promoción y plena realización de los derechos de las personas migrantes y sus familias.

5. Consideración explícita de la migración en los programas de desarrollo sustentable con base en la noción de bienestar, que afinca las estrategias de combate a la pobreza en los principios de inclusión e igualdad, y se orienta a la plena realización de los derechos económicos, sociales, culturales y ambientales.

6. Aumento en la representación y representatividad de las comunidades de personas migrantes mediante mecanismos de gestión y coordinación de política migratoria integral.

De las seis estrategias listadas previamente, el Plan Nacional de Desarrollo (PND 20132018) solo incluye una pequeña parte de esas propuestas, evadiendo reconocer las causas estructurales de la migración internacional y la falta de desarrollo económico nacional para el combate de las causas que han provocado los flujos migratorios internacionales del país.

Queda como antecedente la limitada inclusión de las propuestas en materia migratoria en el PND, pese a que el gobierno mexicano en 2013 promovió -mediante la colaboración de la Secretaría de Relaciones Exteriores, la Secretaría de Gobernación y las organizaciones del Colectivo Plan Nacional de Desarrollo-Migración- varios foros regionales de consulta pública para construir el Programa Especial de Migración 2014-2018 (PEM), en los cuales incluso participaron en su etapa final organizaciones de migrantes de Centroamérica, buscando que en este Programa se integrasen y explicitasen todas las propuestas sobre políticas migratorias del nuevo gobierno. Los objetivos que se plantearon fueron los siguientes: 
1. "Fomentar una cultura de la legalidad y de la valoración de la migración.

2. Promover que las migraciones formen parte de las estrategias de desarrollo regional y local.

3. Impulsar una gestión migratoria eficaz, fundamentada en criterios de facilitación, corresponsabilidad internacional, seguridad fronteriza y derechos humanos.

4. Favorecer los procesos de integración y reintegración de los migrantes y sus familias a las sociedades de origen y destino.

5. Impulsar el acceso a la justicia y la seguridad de las personas migrantes, sus familias y quienes los asistan. (Secretaría de Gobernación [SEGOB]”, 2014, pp. 23-26).

Cada objetivo del PEM cuenta con una serie de estrategias, de las cuales aquí solo señalamos diez:

1. "Fortalecer la incorporación de la migración en la Agenda Global de Desarrollo. En este tema crucial se destacaba la necesidad de que el Gobierno mexicano superara la contradicción de que en los foros internacionales de migración y desarrollo y en espacios de las Naciones Unidas se manifestaba a favor de políticas de migración articuladas con el desarrollo y los derechos humanos, mientras en los hechos seguía con políticas migratorias con enfoque de seguridad nacional.

2. Promover que los programas de desarrollo económico y social incorporen el tema migratorio para favorecer el desarrollo local. Esto resulta imposible cuando se excluyen a los migrantes y sus comunidades de origen del diseño y la aplicación de las políticas públicas sectoriales y regionales.

3. Fortalecer la vinculación entre las personas migrantes mexicanas y sus comunidades de origen o residencia habitual para aprovechar todo el potencial económico, social y cultural para el fortalecimiento de las comunidades transnacionales y su empoderamiento en origen y destino.

4. Impulsar esquemas de migración y movilidad internacional a favor del desarrollo y con pleno respeto a los derechos de las personas migrantes.

5. Diseñar e impulsar acciones para la integración económica, social, cultural y política de las personas migrantes y sus familias.

6. Facilitar y promover el desarrollo educativo de las personas migrantes y sus familias para favorecer su integración y desarrollo personal.

7. Reducir el costo y promover el aprovechamiento productivo de las remesas de las personas migrantes para el desarrollo local y regional.

8. Facilitar y promover la salud integral con criterios diferenciados para las personas migrantes y sus familias.

9. Promover la inserción laboral de las personas migrantes a partir del reconocimiento efectivo de sus derechos, capacidades, habilidades y perfiles diferenciados.

10. Revisar y fortalecer los esquemas para el reconocimiento de los derechos y garantías laborales de los migrantes.” (SEGOB, 2014, pp. 26-35). 
Pese al esfuerzo colectivo y el gran avance en objetivos y propuestas presentadas, el PEM, publicado en el Diario Oficial de la Federación el 30 de abril de 2013, presentó dos enormes limitaciones: Por un lado, la ausencia de una visión integral y transversal de políticas de desarrollo económico que aborden la migración y los derechos humanos para poder incidir en las causas estructurales de los movimientos de población; y por otro lado, el carácter no vinculante del Programa, que carece de obligatoriedad de aplicación federal y estatal, y no cuenta con el respaldo presupuestario y técnico necesario. Lo anterior exhibe el desdén gubernamental expresado a nivel presupuestal cuando en 2015 se asignaron al PEM 50 millones de pesos mexicanos, apenas el $0,19 \%$ de lo recaudado en remesas familiares de los migrantes en ese año.

Paradójicamente, frente a la marginalidad presupuestaria y del tema migratorio en la agenda del Gobierno mexicano, durante las reuniones del $68^{\circ}$ período de sesiones de la Asamblea General de las Naciones Unidas, "Diálogo de Alto Nivel sobre la Migración Internacional y el Desarrollo: "Conseguir que la migración funcione",, sostenidas en octubre 2013, de los 34 puntos de su declaración final, seis fueron aportes de las organizaciones migrantes y de la sociedad civil mexicana:

1. "Reconocer la importancia de la migración internacional en el desarrollo de los países de origen, tránsito y destino, razón por la cual se requieren políticas integrales y coherentes que integren las dimensiones económicas, sociales, ambientales y de derechos humanos.

2. Reconocer el aporte de los migrantes y de las migraciones al desarrollo de los países de origen, tránsito y destino, así como las complejas relaciones entre migración y desarrollo que obligan al diseño de políticas públicas que reconozcan su peculiaridad.

3. Integrar un enfoque de derechos humanos en las políticas de migración y desarrollo modificando los marcos normativos y el funcionamiento de las instituciones responsables de las nuevas políticas públicas.

4. Que todos los países de origen, tránsito y destino asuman el compromiso del diseño y aplicación de las nuevas políticas públicas sobre migración, desarrollo y derechos humanos.

5. Que todos los países colaboren en resolver el reto de las migraciones irregulares para construir una estrategia conjunta que permita establecer estrategias que garanticen migraciones regulares, ordenadas y seguras.

6. Se ratifica la necesidad de promover y proteger los derechos humanos y las libertades fundamentales de todas las personas migrantes, en particular, niños y mujeres, con un enfoque integral, la cooperación y corresponsabilidad internacional” (ONU, 2013, pp. 1-5).

Al final de la elaboración del PEM, pese a sus dos limitaciones centrales, se consideraba que las organizaciones migrantes y de la sociedad civil por lo menos habían obtenido tres productos muy valiosos: una amplia alianza de redes de organizaciones sociales transnacionales; una agenda transnacional compartida, con visión estratégica de desarrollo integral, migración y seguridad humana; y un proceso de creciente aprendizaje, 
colaboración y apoyo transnacional entre las organizaciones sociales y las comunidades de origen y destino de los migrantes con múltiples aliados (García, 2009).

Los integrantes del Colectivo Plan Nacional de Desarrollo-Migración, incrementado con varias organizaciones sociales de Centroamérica y Estados Unidos, decidieron en la última semana de marzo de 2014 cambiar su nombre a Colectivo Migraciones para las Américas (COMPA) (Centro de Análisis e Investigación Fundar, 14 de abril de 2015). Con esta nueva denominación, continúan con sus actividades en defensa y protección de los migrantes, estrategias de incidencia ante el gobierno mexicano, estudios e investigaciones sobre las tendencias migratorias en la región y diseñando propuestas de política pública migratoria con enfoque de derechos humanos.

Resalta la enorme incoherencia en la actuación del Gobierno mexicano que, frente a la gran importancia y aporte de los migrantes mexicanos al funcionamiento del país y sus evidentes cinco dimensiones de flujos poblacionales, no ha promovido los cambios institucionales para atenderlas de forma adecuada mediante la promulgación de políticas públicas respectivas. Frente al mayor protagonismo de los flujos de población de retorno provenientes de Estados Unidos y de transmigrantes de Centroamérica, se sigue sin avanzar en el diseño de políticas públicas que se requiere para gestionar un manejo regular, ordenado y seguro de los migrantes, tal como se propone en los foros internacionales celebrados en los últimos años. Desafortunadamente, persiste una política de seguridad nacional hacia los migrantes en tránsito por México y una política asistencialista y corporativa hacia las organizaciones migrantes mexicanas en Estados Unidos, junto con apoyos escasos y fragmentarios a los migrantes mexicanos retornados, y se constata un abandono de los más de 40 mil desplazados por violencias criminales y megaproyectos en el país.

Desde 2016, con el proceso electoral presidencial en Estados Unidos, Donald Trump inició su guerra económica y política contra México y Centroamérica, colocando a los migrantes mexicanos, las fronteras y sus exportaciones como un peligro para la seguridad nacional de su país. Ante esto, el Gobierno de México no ha sido capaz de diseñar una propuesta de corresponsabilidad de los flujos migratorios regionales entre Estados Unidos, México y Centroamérica bajo una estrategia de desarrollo económico integral y con enfoque de derechos humanos.

El discurso de López Obrador en materia migratoria se ha centrado en sus propuestas de diálogo y colaboración respetuosa con Estados Unidos, poniendo como ejemplo los avances en el llamado Tratado de Libre Comercio México, Estados Unidos y Canadá (T-MEC) y la promoción de los megaproyectos económicos en el sur del país, mediante los cuales -según él- se podrán generar suficientes empleos para mexicanos y migrantes temporales de Centroamérica. En materia migratoria, en el Congreso se señala que el T-MEC es utilizado por Trump para presionar a México y obligarlo a frenar los flujos de indocumentados en la frontera sur. Amenaza el mandatario estadounidense con que de no detenerlos, esto 
afectaría el crecimiento económico del país. Su amedrentamiento dio como resultado que el Gobierno mexicano se comprometiera "a registrar y controlar las entradas en la frontera", así como "a desplegar a la Guardia Nacional por todo el territorio y en especial en la frontera sur" (Gaceta del Senado, 2019, párr. 18). Aragonés (24 de diciembre de 2019) señala que a pesar del escrutinio laboral que en el marco del T-MEC se aplicara a las empresas, sobre todo a las exportadoras para que cumplan con las condiciones de seguridad y salarios, se mantendrán las profundas desigualdades de la sociedad mexicana, pues no hay proyectos en México para superar las diferencias salariales y mejorar las condiciones laborales de los mexicanos. Bajo esas premisas es previsible que no se modifiquen las tendencias migratorias de los últimos años con una marcada tendencia hacia la baja de la migración mexicana y una tendencia creciente de la migración de Centroamérica.

Por su parte, González-Arias (13 de junio de 2019) afirma que la política migratoria de México se plantea bajo dos lógicas. La primera, bajo una lógica de racionalidad instrumental, en la que están presentes las necesidades de las personas migrantes de transitar por territorio mexicano con destino a Estados Unidos, lo que favorece su utilización por el Gobierno estadounidense como moneda de negociación en términos políticos, económicos y hasta sociales, a cambio del endurecimiento de controles migratorios en ambas fronteras de México y así lograr contener los flujos migratorios de indocumentados procedentes principalmente de Centroamérica y del interior del país. La segunda, bajo la lógica de lo apropiado, que refiere a que la posición de México se corresponde con la de un Estado miembro de la sociedad internacional y, en consecuencia, está suscrita a los derechos humanos. Desde estas dos lógicas, González Arias (13 de junio de 2019) sostiene que "México plantea su política migratoria desde la lógica de la racionalidad instrumental, por lo tanto, el Estado hace lo que conviene, aunque ponga en riesgo el bienestar de la sociedad" (párr. 11).

En ese tenor, Donald Trump ha presionado para que México acepte actuar como "tercer país seguro" de los migrantes deportados en proceso de solicitud de trámites de asilo político, al tiempo que amenaza a los países centroamericanos con quitarles la ayuda financiera si no frenan tales flujos migratorios. Para Durand (2018), este éxodo migrante representa un nuevo patrón migratorio de Centroamérica, caracterizado por: migración masiva, en varias caravanas; visible y mediático; con un componente muy importante de niños, jóvenes y familias como nuevo patrón migratorio; espontáneo y organizado, con redes sociales y voceros. Todos esos rasgos son, según Durand, reflejo de una profunda crisis económica, social, política, institucional y de sobrevivencia en Centroamérica, especialmente en Honduras, Guatemala y El Salvador, que cuestiona las estructuras económicas, sociales e institucionales de toda la región, incluido Estados Unidos y sus diversas formas de intervención en la misma, como causa de tales flujos migratorios y cuyos efectos impactan el sistema migratorio en su conjunto. 
Para Jorge Santibáñez Romellón (28 de noviembre de 2018), la presencia de las caravanas migrantes en México ha demostrado la incapacidad del Gobierno mexicano para negociar con Estados Unidos y el presidente Trump. Él señala cómo -durante su paso por el país- la caravana ha generado reacciones contradictorias de apoyo y de rechazo, y Trump en Estados Unidos la ha aprovechado para atacar a los demócratas por su falta de apoyo para la construcción del muro y para adoptar medidas extremas contra los migrantes. En ese contexto señala este autor que sería ideal la colaboración entre ambos países para una gestión migratoria conjunta de los flujos de migrantes, con un enfoque de derechos humanos, pero, lamenta que el Gobierno mexicano haya aceptado unilateralmente recibir a todos los migrantes centroamericanos expulsados de aquel país mientras se procesa su solicitud de asilo político. Plantea Santibáñez Romellón que México perdió la oportunidad de negociar los fondos para asumir los gastos de ese nuevo flujo de retorno de Estados Unidos y los fondos para el desarrollo de las zonas fronterizas donde se asientan esos nuevos "retornados". Sin embargo, sostiene que hay enormes espacios de oportunidad para hacer mejor las cosas en el campo de la gestión de los flujos migratorios entre ambos países.

En otra columna periodística, titulada "López Obrador y la migración centroamericana", Santibáñez Romellón (5 de diciembre de 2018) critica lo débil y superficial del acuerdo internacional firmado por el nuevo Gobierno mexicano con Honduras, Guatemala y El Salvador para promover un Plan de Desarrollo Regional que impulse el crecimiento económico y el empleo, y que incida en las causas estructurales de la migración. El columnista señala las limitaciones centrales de dicho acuerdo: la grave ausencia de Estados Unidos en el acuerdo, teniendo en cuenta que este país es el eje del sistema migratorio regional y fuente de muchas de las causas estructurales de los flujos migratorios; la pobreza y falta de empleos; la violencia e inseguridad generadas fundamentalmente del éxodo centroamericano; sin mencionar las responsabilidades, funciones y compromisos sobre ambos factores de los países de Centroamérica, y el desfase de la información migratoria que maneja el presidente mexicano Andrés Manuel López Obrador (AMLO). Con prudencia, Santibáñez afirma que este es apenas el inicio del nuevo gobierno. Al respecto señalamos que concedamos el beneficio de la duda y sobre todo contribuyamos para que en el desgarrador proceso de salida de los países del llamado Triángulo del Norte, México juegue un liderazgo positivo para los centroamericanos y no solo para sus gobernantes.

Como indicamos antes, desde la campaña presidencial, AMLO reiteraba la necesidad de un gran plan de desarrollo económico para Centroamérica para incidir en las causas estructurales de la migración internacional de pobreza y ausencia de empleo, con financiamiento de Estados Unidos, México, Canadá y los países de esa región. El problema radica en que no existe ninguna propuesta equivalente para las zonas de alta migración internacional de México - pese a los proyectos planteados por diversos de organizaciones sociales, migrantes y de académicos en los últimos veinte años-, no existe ningún proyecto concreto para la región mencionada, no hay ningún compromiso de financiamiento para ese 
objetivo, y en el acuerdo mencionado se excluye al país más importante del sistema migratorio regional.

A inicios de 2019, un nuevo flujo migratorio de Centroamérica anunciaba y realizaba su ingreso a México con AMLO al frente del nuevo gobierno y denotando algunos rasgos diferentes respecto a la experiencia previa de los flujos de octubre y noviembre: el nombramiento del Dr. Tonatiuh Guillén -distinguido investigador social al frente del Instituto Nacional de Migración-, que anunciaba una reorientación de la visión de seguridad pública, centrada ahora en la gestión migratoria con un enfoque de derechos humanos; el ingreso regulado y ordenado al país mediante el otorgamiento de una visa temporal a cerca de 13 mil migrantes y el esfuerzo de colaboración de los tres niveles de gobierno: ayuntamientos, gobiernos estatales y gobierno federal, para acompañar institucionalmente de mejor manera a ese flujo durante su tránsito hacia la frontera norte.

La mayor parte de este flujo llega a Piedras Negras, Coahuila, y ante el muy limitado acceso a los trámites de asilo político en Estados Unidos, su permanencia en la frontera norte de México se ha convertido en un factor de tensión institucional entre los gobiernos locales y el gobierno federal. Esta situación ha obligado a que el nuevo Gobierno acelere la lucha contra la corrupción en el Instituto Nacional de Migración y promueva otros cambios normativos e institucionales para hacer frente a la gestión de ese flujo, su estancia y demandas de empleo y servicios sociales en México ante las nuevas y crecientes solicitudes de ingreso de transmigrantes en la frontera sur.

Al igual que en las caravanas de los meses previos, la de inicios de 2019 generó la reactivación discursiva de Trump en Estados Unidos. En la visión del mandatario, estas constituían un nuevo peligro para las fronteras y la seguridad de su país, lo que lo llevó a fortalecer su lucha en el Congreso por conseguir mayores fondos para la construcción de un muro y lanzar la campaña para su reelección en 2020. En tanto en México, se destacaba la capacidad de colaboración de diversas organizaciones de la sociedad civil de las diferentes regiones del país por donde pasó la caravana, en contraposición de la falta de apoyo financiero y técnico del gobierno federal para poder enfrentar la llegada y estancia de los 13 mil integrantes de la caravana de 2019.

A nivel del gobierno federal, ante las declaraciones del presidente de Estados Unidos y frente a las reacciones en México de los flujos de transmigrantes de 2019, los secretarios de Relaciones Exteriores, Marcelo Ebrad, y de Gobernación, Olga Sánchez Cordero, expresaron que México contaba con una nueva política migratoria que se basaba en una estrategia de no contención y respeto de los derechos humanos (Durand, 2018). Por su parte, el subsecretario de Gobernación, Alejandro Encinas, señaló que la política migratoria es de Estado y responde estrictamente a los intereses de México; por lo tanto, el subsecretario hizo énfasis en el hecho de hacer su propia política migratoria: ordenada, regulada y segura, basada en el pleno ejercicio de la soberanía de México. 
Tanto Ebrad como Sánchez Cordero afirmaron que en México se terminaría con la política de criminalización al migrante y se haría una reestructuración "desde sus entrañas" al Instituto Nacional de Migración para cambiar esta visión. La política migratoria del nuevo Gobierno, según, ambos funcionarios, estaría regida por dos ejes: el respeto y la promoción de los derechos humanos y el desarrollo social y económico de las regiones expulsoras, junto con trabajar de forma estrecha con la sociedad civil. Olga Sánchez destacó el nuevo diálogo que se estaba dando con los países de Centroamérica en temas migratorios y la renovación de las relaciones con Estados Unidos, con el que por primera vez en la historia se llegó a un acuerdo que abarca las causas de la migración y no solo políticas de contención de este fenómeno.

El 13 de marzo de 2019, el secretario de Relaciones Exteriores, Marcelo Ebrard, anunció el decálogo para apoyar a migrantes mexicanos en Estados Unidos, documento que busca empoderar a la comunidad mexicana y concretar la ampliación de programas de educación, salud y deportes (Muñoz y Urrutia, 1 de marzo de 2019). En general, se trata de medidas que apuntan a brindar mayor información sobre los programas del Gobierno mexicano hacia los migrantes, mayor colaboración entre las propias dependencias del Gobierno, mejorar el funcionamiento de esas dependencias en el exterior con mayor eficiencia y enfoque de derechos humanos, aumentar la defensa legal de los migrantes mexicanos en el exterior y fortalecer la cultura nacional. Prácticamente, no hay nada nuevo, tampoco acciones concretas hacia la enorme comunidad en Estados Unidos y, menos, el anuncio de mayores recursos humanos, técnicos y financieros que posibiliten esos diez objetivos.

El 28 de febrero en Washington, Olga Sánchez Cordero, secretaria de Gobernación, en la conferencia "Una nueva política migratoria para una nueva era" ratificó la nueva visión de la política migratoria del Gobierno mexicano, que reconoce que las causas de las migraciones son la necesidad, la pobreza, la falta de empleo y las violencias, lo cual implica incidir en sus causas estructurales y garantizar una gestión migratoria en la región con enfoque de derechos humanos. Bajo esa nueva visión se erradica la estigmatización hacia los migrantes de Centroamérica como delincuentes, ya que solo el $1 \%$ tiene antecedentes penales. Sin embargo, se hace necesario reconocer que el monto actual de inmigrantes se puede duplicar a 700 mil migrantes irregulares anuales por la frontera sur de México, con 370 puntos de ingreso irregular, los que reflejan una auténtica catástrofe humanitaria en la región, la que no puede resolverse solo con la política migratoria de un país, por lo que se requiere del apoyo logístico y financiero internacional, como sucede en los campos de refugiados construidos en Turquía y Líbano, sin olvidar la degradación de las condiciones de vida de quienes viven en esas instalaciones construidas de forma emergente.

Sánchez Cordero resaltó en su gira a Washington ante la secretaria de Seguridad Nacional de Estados Unidos, Kirjsten Nielsen, funcionarios del Banco Interamericano de Desarrollo e investigadores del Instituto de Política Migratoria, que México no es la "causa" del incremento migratorio de centroamericanos hacia aquella nación y, por el contrario, busca 
ser parte de la solución a partir de promover una migración ordenada y segura, con base en el respeto a los derechos humanos de los extranjeros mediante una nueva política migratoria que no trata de contener, detener y deportar, sino de registrar y regular el flujo de personas basándose en el respeto de los derechos humanos como eje.

Desde el verano de 2018, una vez que las elecciones presidenciales de forma abrumadora dieron el triunfo al Movimiento de Regeneración Nacional (Morena) y López Obrador, quedando estos como responsables del Gobierno mexicano, diversas organizaciones de la sociedad civil, integrantes del COMPA, incrementaron sus convocatorias y reuniones de trabajo con otras numerosas organizaciones y sectores para colaborar en el diseño de un nuevo marco normativo para los flujos de movilidad poblacional en México y de nuevas políticas migratorias estatales, que adopten una visión integral y transversal y con un enfoque de derechos humanos.

Dentro de estas organizaciones -las que durante más de diez años han estado trabajando en el apoyo y defensa de múltiples sectores de migrantes en México y en el diseño de políticas migratorias con enfoque de derechos humanos, desarrollo, migración, cambio institucional y temas afines- destaca el Grupo de Trabajo sobre Política Migratoria (GTPM), que promueve diversas acciones en conjunto con el Poder Legislativo, y cuenta con múltiples organizaciones sociales de migrantes, organizaciones religiosas y académicas para colaborar en la construcción de las propuestas antes mencionadas.

En febrero de 2019, el GTPM dio a conocer su propuesta "Hacia una política migratoria de respeto a los derechos humanos en México", en la cual expresa que a pesar de los avances en marcos normativos y políticas públicas sobre derechos humanos y migración, como fue la reforma constitucional en materia de derechos humanos y la promulgación de la Ley de Migración y de la Ley sobre Refugiados, Protección Complementaria y Asilo Político, y sus respectivos reglamentos, aún existen dos retos centrales en su aplicación. Por un lado, la política migratoria en México debe plantear marcos normativos y políticas públicas con perspectiva de seguridad humana que garanticen los derechos humanos de las personas en diferentes contextos de movilidad y de sus familiares. Entre otras cosas, eliminando la detención de personas migrantes en estaciones migratorias como práctica generalizada, así como la deportación expedita sin la evaluación individual de cada caso que permita identificar necesidades de protección debido a las circunstancias por las que las personas emigraron o la ponderación de los derechos sociales como el derecho humano al trabajo, la seguridad social, salud, vivienda entre otros. Por otro lado, cada Secretaría de Estado y entidad en los tres niveles de gobierno deberá cumplir con su mandato de respeto a los derechos humanos de las personas migrantes, partiendo del principio de no discriminación, y el Instituto Nacional de Migración (INM) debería ser una entidad que opere los procedimientos administrativos de manera eficiente y con apego irrestricto a los derechos humanos. En términos generales, esto significa para el GTPM (Martínez, s. f.) lo siguiente: 
- Diseñar y poner en marcha una política de Estado que apueste a la seguridad humana, asegurando la parte programática que permita contribuir a garantizar el goce efectivo de los derechos humanos de personas en contextos de migración internacional en México.

- Armonizar el marco normativo en México a nivel federal y estatal, bajo los más altos estándares de protección de derechos humanos, tomando en cuenta diversas recomendaciones de órganos de las Naciones Unidas y el Sistema Interamericano de Derechos Humanos.

- Diseñar de la mano con la sociedad civil, agencias de Naciones Unidas y organismos públicos de derechos humanos, mecanismos de rendición de cuentas, participación ciudadana y transparencia.

- Garantizar el acceso a la justicia a personas víctimas de delitos y de violaciones a derechos humanos y el establecimiento de garantías de no repetición.

Durante el mes de febrero 2019 surgió una muy importante propuesta de política migratoria para el nuevo Gobierno mexicano de parte de numerosas organizaciones civiles integrantes del COMPA, que como indicamos antes, llevan más de diez años trabajando los temas de migración internacional, protección, derechos humanos y otros. Dicha propuesta, elaborada por Karina Arias, Rodolfo Córdoba y Alexandra Délano (2019), con el título de "Personas migrantes y refugiadas como prioridad para el Gobierno: un sistema integral para personas en movilidad en México", en su primera parte resalta las múltiples causas estructurales de la migración; las cuatro dimensiones migratorias de México de origen, tránsito, destino y retorno (no incluyen los desplazamientos internos); y la falta de voluntad política del Gobierno mexicano por más de cuatro décadas para construir un verdadero sistema desde el ejecutivo federal para coordinar de forma transversal con todas las dependencias y niveles de gobierno las nuevas políticas migratorias que se requieren para responder adecuadamente a las diferentes dimensiones de ese fenómeno en el país hoy.

Bajo los señalamientos anteriores, los autores plantean el reto de que México se posicione a nivel mundial mediante la adopción de cambios institucionales en políticas migratorias relevantes de acuerdo con las propuestas de avanzada que se han planteado en los diferentes foros mundiales sobre el tema, aportando una visión integral, transversal y de largo plazo. Este cambio institucional coincide con los señalamientos del nuevo presidente de México, Andrés López Obrador, de diseñar políticas de cambio estructural, de desarrollo integral, que incidan en las causas de fondo de la migración y que hagan viable a mediano plazo el derecho a no emigrar o a hacerlo como alternativa y no como necesidad, como sucede hasta ahora.

Arias, Córdoba y Délano (2019) proponen una nueva visión migratoria con los siguientes rasgos: integralidad, derechos humanos, género, transversalidad, justicia, desarrollo sustentable, cultura de inclusión, hospitalidad y apertura intersectorial, transnacional y 
regional, y transexenal. Para hacer viable su propuesta, ellos plantean la creación de una estructura de coordinación de alto nivel institucional llamada Sistema Integral para Personas en Movilidad (SIPM), que articule todas las dependencias del Gobierno mexicano y los poderes de la Unión con un Consejo de Estado multiactor a nivel de Estado en materia de movilidad humana, con las siguientes características:

1. El Sistema debe ser convocado desde la Oficina de la Presidencia de la República. El presidente estaría al frente del Consejo de Estado multiactor, cuya Secretaría Técnica estará a cargo de la Unidad de Política Migratoria (UPM), ahora ubicada en la Subsecretaria de Población, Migración y Asuntos Religiosos, de la Secretaria de Gobernación, pasando a la Oficina de la Presidencia, elevando así sus capacidades y mandato.

2. Lo anterior permite construir y establecer un nuevo Programa Nacional de Movilidad Humana con la participación de todos los actores institucionales y de la sociedad civil transnacional vinculados con la movilidad humana en México bajo sus diferentes dimensiones.

3. El Sistema debe contar con un Consejo de Estado multiactor en movilidad humana que retomaría el Consejo Consultivo de Política Migratoria, ubicado hasta ahora en la Secretaria de Gobernación, ampliado con nuevos actores, propuestas y potenciales. Este Consejo se propone como el verdadero motor articulador, promotor y coordinador de la materialización de la nueva política de movilidad humana, al cual se le asignan ocho funciones estratégicas de vinculación permanente con los organismos internacionales de migración y derechos humanos, la negociación de acuerdos internacionales sobre movilidad laboral con derechos plenos, la promoción de cambios normativos, la creación del sistema de desempeño y la evaluación presupuestal, la articulación permanente con las secretarías del gobierno mexicano, la Comisión de Asuntos Migratorios (CONAGO) y la Coordinación Nacional de Oficinas Estatales de Atención a Migrantes (CONOFAM).

4. Es necesario realizar cambios puntuales en la estructura institucional actual y las funciones que desempeñan instancias como la Unidad de Política Migratoria, el Consejo Nacional de Población (CONAPO), el cambio de la Comisión de Ayuda a Refugiados (COMAR) a Instituto Mexicano de Ayuda a Refugiados (IMAR), y el Instituto Nacional de Migración (INM o INAMI). Se propone, además, modificar funciones en los Oficiales de Protección a la Infancia, quedando a cargo de la Procuraduría Federal para tal fin; el Programa Paisano se ubica en la Dirección General de Protección a los Mexicanos en el Exterior de la Secretaría de Relaciones Exteriores, y los Grupos Beta se dispone que sean incluidos como un programa de Protección Civil. Asimismo, se plantea que tanto el INM como el IMAR dependan de la Subsecretaría de Población, Migración y Asuntos Religiosos de la Secretaria de Gobernación. 
En el documento señalado, Arias, Córdoba y Délano (2019) proponen 19 acciones prioritarias para los primeros 120 días, al expresar que el Consejo de Estado multiactor impulsaría, a través del sistema, acciones y reformas institucionales y normativas por sector y dependencia de conformidad con la nueva visión migratoria, comenzando en los primeros 100 días de gobierno, que respondan a cada una de las dimensiones migratorias de México. Queda claro a cualquier lector la importancia de esta propuesta de política pública sobre movilidad humana en México, que generó un gran debate y lucha política nacional en los foros públicos, el Congreso y el Senado sobre la problemática actual de México, respecto del modelo de país, de las instituciones y de las políticas gubernamentales que rijan el futuro de México. Esta lucha se expresó desde diciembre de 2018 en el debate presupuestal para 2019, con la "guerra" contra el robo masivo de petróleo, las pugnas con el Poder Judicial por las enormes ganancias, y la creación de la Gendarmería en el país. Todo este proceso se ha visto complejizado por las presiones por parte de las calificadoras financieras internacionales ante la baja evaluación del crecimiento económico de México durante el periodo 2019-2020, y la narrativa en contra de la inversión pública para rescatar la industria petrolera y eléctrica del país, que de hecho significa un condicionamiento de los grandes inversionistas privados, nacionales y extranjeros para sustentar los megaproyectos regionales del nuevo Gobierno, a no mediar un cambio en la política macroeconómica de austeridad y a favor del capital financiero internacional.

En el discurso oficial, la visión estratégica del actual gobierno (2018-2024) en materia migratoria tiene los siguientes componentes (Gobierno de México, 2020):

1. Responsabilidad compartida. A grandes rasgos contempla

abordar el fenómeno desde diversas facetas, no sólo para atender la movilidad internacional en tránsito hacia Estados Unidos, sino como una oportunidad para impulsar el desarrollo en la región y la movilidad laboral, turística y comercial (...) renovar el diálogo con el Gobierno de Estados Unidos para garantizar la protección de las y los connacionales que se encuentran en territorio estadounidense y de quienes retornan a México, así como de los mexicanos que radican en otras partes del mundo. (Gobierno de México, 2020, párr. 3)

2. Movilidad y migración internacional regular, ordenada y segura. "Es ésta una de las principales tareas del quehacer gubernamental que, por un lado, necesita abrir vías $\mathrm{y}$, por el otro, fortalecer las existentes para permitir a las personas moverse hacia y desde México de manera segura, ordenada y regular" (Gobierno de México, 2020, párr. 7).

3. Atención a la migración irregular: consiste en proporcionar a este grupo medidas de protección a su integridad física y psicológica. Tales acciones deben ser evaluadas, mejoradas y fortalecidas, así como supervisar el trabajo de los Grupos Beta del Instituto Nacional de migración. Impulsar programas de regulación migratoria y flexibilizar los procesos burocráticos. 
4. Fortalecer las capacidades de las instituciones. Este componente busca que las instituciones encargadas de la política migratoria

(como son la Comisión Mexicana de Ayuda a Refugiados, el Instituto Nacional de Migración, las oficinas consulares y la Unidad de Política Migratoria, Registro e Identidad de Personas) analicen y adquieran nuevas habilidades y competencias a través de la promoción de las reformas estructurales necesarias que les permitan vincularse y complementar su actuar para atender el fenómeno migratorio de manera integral, transversal, interinstitucional y con enfoque de derechos humanos y perspectiva de género, así como recabar, procesar y sistematizar de manera periódica y permanente información estadística que provea de elementos para la adecuada toma de decisiones. (Gobierno de México, 2020, párr. 13)

5. Protección de mexicanas y mexicanos en el exterior. El Gobierno, en el reconocimiento de las grandes contribuciones que hacen los mexicanos al país en términos económicos, sociales y culturales,

crea un componente específico para su protección y atención; su vinculación con sus comunidades de origen; los procesos de coordinación con todos los órdenes de gobierno, así como políticas culturales que permitan la continuidad de los vínculos con México, no sólo de forma material sino también simbólica, fortaleciendo el arraigo y el sentimiento de pertenencia a la nación, para lo cual cobra especial relevancia el rol de la Secretaría de Cultura para impulsar políticas y programas acordes. Esto busca no sólo fortalecer la presencia de México en el exterior, sino además incentivar el retorno de personal calificado. (Gobierno de México, 2020, párr.14)

6. Integración y reintegración de personas migrantes. Este componente parte

de la lógica de la inclusión, es decir, cuentan con la participación activa de todos los actores involucrados. Las personas migrantes no sólo son receptoras de las acciones o políticas, sino que tienen una participación activa en su definición, ejecución y seguimiento, ya que a través de diversos ejercicios se les dota de herramientas para que hagan exigibles sus derechos. (Gobierno de México, 2020, párr. 17)

7. Desarrollo sostenible en comunidades migrantes:

Este componente busca satisfacer las necesidades actuales de las personas en comunidades expulsoras y receptoras de migrantes, particularmente en la región sur-sureste, sin comprometer los recursos y posibilidades de las futuras generaciones, con la finalidad de lograr la autosuficiencia regional, asegurar que la actividad económica mejore la calidad de vida de todas las personas, usar los recursos eficientemente, promover el máximo de reciclaje y reutilización, así como emprender otras acciones que mantengan y mejoren el sistema ambiental a través del diseño de políticas públicas y de combate al rezago social, orientadas a reducir los niveles de marginación. (Gobierno de México, 2020, párr. 23) 
Para sostener cada uno de estos componentes de la actual política migratoria es fundamental el trabajo coordinado y articulado en los tres órdenes de gobierno, organismos internacionales, organizaciones de la sociedad civil e iniciativa privada, así como promover campañas permanentes de difusión de los derechos de las personas migrantes, sus contribuciones en las comunidades receptoras mediante el intercambio de habilidades y competencias, y el enriquecimiento cultural que la migración genera entre países. (Gobierno de México, 2020, párr. 24).

Durante el segundo semestre de 2018 -tiempo en que López Obrador es electo- y en el transcurso de su primer año de gobierno (2019), resalta la contradicción entre los discursos progresistas oficiales sobre políticas migratorias y la persistencia en los hechos de una política de seguridad nacional y contención de los flujos migratorios de Centroamérica. Por ello, el GTPM invitó reiteradamente al Gobierno de turno a ser congruente en su postura, acciones y discurso sobre la política migratoria y la urgencia de garantizar los derechos humanos de los migrantes, subrayando que estos no podían subordinarse a los intereses económicos, políticos y comerciales en el marco de las relaciones internacionales (Voces mesoamericanas, 2019).

Tal como señala Cecilia Bobes (2019), la acción militar para frenar la migración que cruza territorio mexicano, conlleva un retroceso en la política migratoria del país y debilita la estrategia planteada basada en derechos humanos, profundizando así las brechas entre el marco normativo, la política y la gestión de la migración. Los hechos ocurridos en materia migratoria, ampliamente documentados, que responden a las demandas del Gobierno de Estados Unidos para frenar la migración (operativos, redadas, retenes de verificación, detenciones y deportaciones masivas, violación al derecho de libre tránsito) no solo ponen en entredicho las siete componentes de la visión estratégica del Gobierno actual en materia migratoria, sino que su aplicación y resultados dependen de lo que vaya dictando el Gobierno estadounidense. Por lo que reiteramos la necesidad de incluir las demandas de los migrantes en la agenda de la política del Gobierno (en sus tres órdenes) y fortalecer su vínculo con la diáspora en lugar de debilitarlo, como ocurre hasta el momento, al dejarlos fuera del presupuesto y obviar las demandas y propuestas de los clubes de migrantes y la sociedad civil en favor de los migrantes mexicanos en el exterior y de aquellos que cruzan el territorio mexicano. Nos sumamos al planteamiento de González-Arias (2019), quien afirma que la política migratoria debe pasar de la lógica instrumental a la lógica de lo apropiado. Hay claridad de lo que hay que hacer, pero no existe voluntad política del Gobierno, de los partidos político y del Congreso para hacer los cambios necesarios. 


\section{Conclusiones}

No se puede entender el funcionamiento de México en los últimos cincuenta años con profundas crisis económicas y sociales sin considerar la importancia que ha tenido la migración internacional a Estados Unidos y la creciente interdependencia económica, comercial, social y geopolítica entre ambos países. En efecto, el crecimiento explosivo de la migración mexicana a aquel país, desde fines de la década de 1970 hasta el estallido de la Gran Recesión en el periodo de 2007-2008, refleja los impactos profundos de esas crisis en la estructura productiva y la capacidad de generación de empleos en México con una creciente interdependencia laboral de Estados Unidos en determinados mercados en la agricultura, servicios de alimentación, hospedaje, construcción, jardinería, salud y cadenas de cuidado.

Tal proceso migratorio hasta el estallido de la crisis financiera resultó benéfico para ambos países. Para México reducía las tensiones del raquítico mercado laboral, generaba montos crecientes de remesas para el país y posibilitó el surgimiento de cientos de organizaciones comunitarias transnacionales con importantes impactos en sus comunidades de origen que realizaban inversiones solidarias migrantes. Por su parte, Estados Unidos se benefició con un enorme "ejercito migrante de reserva" para sectores laborales caracterizados por los bajos salarios, la precarización, la ausencia de seguridad social y la vulnerabilidad, pero necesarios para la economía de ese país.

Hasta el estallido de la crisis financiera en Estados Unidos en 2007-2008, los gobiernos de México y Centroamérica de forma imprudente creían que toda la vida podrían seguir mandando migrantes a aquel país y que no había necesidad de aplicar políticas de desarrollo económico nacional y de generación de empleo. Con la crisis y sus impactos de desplome de los flujos migratorios y de las remesas, y ante el cuestionamiento institucional que surgió respecto a la posibilidad de reintegrar el flujo creciente de migrantes retornados y deportados por las políticas xenófobas y de militarización de la frontera norte, se presentó la oportunidad para que los gobiernos de la región analizaran diversas propuestas de nuevas políticas públicas de desarrollo económico, migración y derechos humanos, que desde fines de la década de 1990 diversas organizaciones transnacionales (migrantes, investigadores, ONG, iglesias) venían haciendo en México, Centroamérica y Estados Unidos. Sin embargo, dicho ejercicio de análisis y construcción de nuevas políticas públicas de desarrollo y migración no se hizo y se aplicó la misma estrategia de seguridad nacional sobre las migraciones que la establecida por Estados Unidos desde los acontecimientos de septiembre de 2001, pero ahora incluso con mayor fuerza.

En el caso de México, la falta de interés del Estado y de la clase política sobre sus migrantes, sus problemas, demandas y propuestas, refleja una enorme incoherencia frente al aporte migrante al país por más de cuatro décadas y frente a una gran cantidad de propuestas de políticas y programas migratorios hechos en el país y en Estados Unidos por 
cientos de organizaciones migrantes. Es cierto que por vez primera en el Plan Nacional de Desarrollo 2013-2018 se introduce el tema brevemente y se trabaja posteriormente en la elaboración del Programa Especial de Migración, que es la propuesta más avanzada sobre política migratoria, pero sin ser vinculante y sin tener el carácter de obligatorio para los tres niveles de gobierno, careciendo entonces del apoyo jurídico, técnico y financiero necesario para su aplicación. En el contexto anterior de marginalidad de los migrantes, la movilidad humana en territorio mexicano se complejiza en sus dimensiones, como espacio de origen, tránsito, destino, retorno, desplazamientos, asilo y refugio ante el incremento masivo de migrantes procedentes de Centroamérica.

La economía mexicana creció lentamente por debajo del 2\% desde el 2012 hasta el 2018, la deuda pública y el desempleo aumentaron, se redujo la migración hacia Estados Unidos, pero paradójicamente subieron las remesas y los flujos migratorios centroamericanos. Sin embargo, el nuevo gobierno no promueve ninguna propuesta de política migratoria y de desarrollo que responda a esas nuevas dimensiones de la movilidad humana en el país, sino que persiste en la visión de seguridad nacional sobre las migraciones en general, y en el asistencialismo y corporativismo hacia los migrantes mexicanos.

El principal desafío de las políticas migratorias para el nuevo gobierno de México, que rige para el periodo 2018-2024, es mantener la coherencia en relación con el discurso que enarbola, cuando plantea que la alternativa es el desarrollo económico y el establecimiento de políticas migratorias con enfoque de derechos humanos, pero que en la práctica contrariamente sigue aplicando la misma política neoliberal de austeridad y la política de seguridad nacional. La coherencia radica en establecer una verdadera estrategia de desarrollo económico nacional que promueva y diseñe políticas proactivas sectoriales, regionales y de empleo, articuladas con la política migratoria integral con enfoque de derechos humanos, respaldadas con todo el soporte legal, técnico y financiero para su aplicación por los tres niveles de gobierno en el país.

\section{Referencias bibliográficas}

Aragonés, A. M. (24 de diciembre de 2019). El T-MEC y la migración. La Jornada. Recuperado de https://www.jornada.com.mx/2019/12/24/opinion/012a1pol

Arias, K., Córdoba, R. y Délano, A. (2019). Personas migrantes y refugiados como prioridad para el Gobierno: un Sistema Integral para personas en Movilidad en México. Ficha 2. [En línea]. IMUMI. Recuperado de http://pendientesenmigracion.imumi.org/wp-content/uploads/2019/07/Ficha-2Sistema-Integral-para-Personas-en-Movilidad-en-M\%C3\%A9xico.pdf

Bobes, C. (2019). De las puertas abiertas al «ya no son bienvenidos» El giro de la política migratoria mexicana. Revista Nueva Sociedad, 284, noviembre-diciembre. 
Recuperado de https://www.nuso.org/articulo/de-las-puertas-abiertas-al-ya-no-sonbienvenidos/

Canales, A. (2012). La migración mexicana ante la crisis económica actual. Crónica de un retorno moderado. Revista Interdisciplinar da Mobilidade Humana REMHU, 20(39), 117-134 Recuperado de http://www.scielo.br/pdf/remhu/v20n39/v20n39a07.pdf

Canales, A. y Meza, S. (2016). Fin del colapso y nuevo escenario migratorio MéxicoEstados Unidos. Migración y Desarrollo, 27, 65-107. Recuperado de: http://www.scielo.org.mx/pdf/myd/v14n27/1870-7599-myd-14-27-00065.pdf

Castillo, M. y Burstein, J. (2014). El desarrollo integral con migrantes. México, Oxfam, APOFAM, Voces Mesoamericanas y Apofam.

Centro de Análisis e Investigación Fundar (14 de abril de 2015). El Colectivo Migraciones para las Américas (COMPA) expresa su preocupación por la seguridad física y jurídica de las personas migrantes que actualmente se encuentran en el albergue Hermanos en el Camino A.C de Ixtepec, Oaxaca. [En línea]. Fundar. Recuperado de http://fundar.org.mx/el-colectivo-migraciones-para-las-americas-compa-expresa-supreocupacion-por-la-seguridad-fisica-y-juridica-de-las-personas-migrantes-queactualmente-se-encuentran-en-el-albergue-hermanos-en-el-cam/

CONEVAL (2020). La crisis sanitaria generada por la covid-19 y sus consecuencias económicas ponen en riesgo avances en desarrollo social y puede afectar en mayor medida a grupos vulnerables. Sitio web CONEVAL. Recuperado de https://www.coneval.org.mx/Evaluacion/IEPSM/Paginas/Politica_Social_COVID19.aspx

Durand, J. (2018). Crisis migratoria, humanitaria, política y mediática. Conferencia magistral, Seminario Internacional de Migraciones, Mazatlán, Sinaloa, México, noviembre.

Gaceta del Senado (3 de julio de 2019). Información Parlamentaria. Gaceta: LXIV/1SR19/97023. [En línea]. Recuperado de https://www.senado.gob.mx/64/gaceta_del_senado/documento/97023

García, R. (2009). Desarrollo económico y migración internacional: los desafíos de las políticas públicas en México. México: Universidad Autónoma de Zacatecas.

García, R. (2010). Migración internacional y desarrollo en América Latina y el Caribe. Del mito a la realidad. En G. Vidal y O. de León (eds.), América Latina: democracia, economía y desarrollo social (pp. 141-160). Madrid: Trama.

García, R. (2019). México. La nación desafiada. Análisis y propuesta ante la migración y la falta de desarrollo en México. México: Miguel Ángel Porrúa-Universidad Autónoma de Zacatecas. 
García, R. y Gaspar, S. (2017). Migración de retorno de Estados Unidos a seis estados de México. Hacía la reintegración familiar y comunitaria. En R. García Zamora (ed.), El retorno de los migrantes mexicanos de Estados Unidos a Michoacán, Oaxaca, Zacatecas, Puebla y Chiapas 2000-2012 (pp. 15-64). México: Miguel Ángel Porrúa y Universidad Autónoma de Zacatecas.

Gaspar, S. (2012). Migración México-Estados Unidos en cifras (1990-2011). Migración y Desarrollo, 10(18), 101-139. Recuperado de http://www.scielo.org.mx/pdf/myd/v10n18/v10n18a4.pdf

Gaspar, S. (2018). Medición de la emigración de México a Estados Unidos, 1950-2016. Región y Sociedad, 30(73), 3-41. Recuperado de https://regionysociedad.colson.edu.mx:8086/index.php/rys/article/view/847/1295

Gobierno de México. (2020). Visión ejecutiva de la política migratoria: Principales componentes. [En línea]. Gobierno de México. Secretaría de Gobernación.

Recuperado de http://portales.segob.gob.mx/es/PoliticaMigratoria/2_Vision_ejecutiva_de_la_politica _migratoria_PRINCIPALES_COMPONENTES/179\#021 [Consultado el 19 de agosto de 2020].

González-Arias, A. (13 de junio de 2019). La política migratoria en México. Foreing Affairs Latinoamérica. Recuperado de http://revistafal.com/la-politica-migratoria-en$\underline{\text { mexico/ }}$

Martínez, C. (s. f.). GTPM promueve cambios legislativos para lograr una política migratoria de respeto a los derechos humanos en México. [En línea]. Sitio web Grupo de Trabajo sobre Política Migratoria. Recuperado de https:/gtpm.mx/gtpmpromueve-cambios-legislativos-para-lograr-una-politica-migratoria-de-respeto-a-losderechos-humanos-en-mexico/

Muñoz, A. E. y Urrutia, A. (1 marzo de 2019). Implanta la SRE decálogo para la atención de los mexicanos en Estados Unidos. La Jornada, p. 11. Recuperado de https://www.jornada.com.mx/2019/03/01/politica/011n1pol

Organización de las Naciones Unidas (ONU) (2013). Declaración del Diálogo de Alto Nivel sobre la Migración Internacional y el Desarrollo. Sexagésimo octavo período de sesiones Tema 21 e) del programa Globalización e interdependencia: migración internacional y desarrollo. Asamblea General. [En línea]. ONU. Recuperado de https://undocs.org/es/A/68/L.5

OIM (2009). The impact of financial crises on international migration: Lessons learned. OIM Migration Research Series $N^{\circ}$ 37. Geneva: OIM. Recuperado de https://publications.iom.int/system/files/pdf/mrs37_en.pdf 
Santibáñez Romellón, J. (5 de diciembre de 2018). López Obrador y la migración centroamericana. La Jornada. Recuperado de https://www.jornada.com.mx/2018/12/05/opinion/027a2pol

Santibáñez Romellón, J. (28 de noviembre de 2018). ¿Negociar con Estados Unidos? La Jornada, Sección Opinión. Recuperado de https://www.jornada.com.mx/2018/11/28/opinion/024a1pol

Secretaría de Gobernación (SEGOB) (2014). Programa Especial de Migración 2014-2018 (PEM). Diario Oficial de la Federación, 30 de abril. Recuperado de https://www.gob.mx/cms/uploads/attachment/file/44579/Plan_Especial_de_Migracio $\underline{\text { n.pdf }}$

Voces mesoamericanas (2019). Impactos de la política migratoria de México en la frontera sur. Hallazgos de la misión de observación de derechos humanos en Tapachula, Chiapas. Recuperado de https://vocesmesoamericanas.org/wpcontent/uploads/2019/11/INFORME-MODH-

MIGRACIO\%CC\%81N_SuresteMe\%CC\%81xico-WEB.pdf 